Revue d'archéologie préhistorique

$23 \mid 2012$

Varia

\title{
Biostratigraphie des niveaux solutréens de Laugerie-Haute (Les Eyzies, Dordogne, France). Implications archéologiques
}

Françoise Delpech

\section{OpenEdition}

\section{Journals}

Édition électronique

URL : http://journals.openedition.org/paleo/2457

DOI : $10.4000 /$ paleo.2457

ISSN : 2101-0420

Éditeur

SAMRA

\section{Édition imprimée}

Date de publication : 15 décembre 2012

Pagination : 105-116

ISSN : $1145-3370$

\section{Référence électronique}

Françoise Delpech, «Biostratigraphie des niveaux solutréens de Laugerie-Haute (Les Eyzies,

Dordogne, France). Implications archéologiques », PALEO [En ligne], 23 | 2012, mis en ligne le 19 avril

2013, consulté le 02 août 2020. URL : http://journals.openedition.org/paleo/2457 ; DOI : https://

doi.org/10.4000/paleo.2457

Ce document a été généré automatiquement le 2 août 2020.

\section{cc) (†)}

PALEO est mis à disposition selon les termes de la licence Creative Commons Attribution - Pas d'Utilisation Commerciale - Pas de Modification 4.0 International. 


\title{
Biostratigraphie des niveaux solutréens de Laugerie-Haute (Les Eyzies, Dordogne, France). Implications archéologiques
}

\author{
Françoise Delpech
}

\section{Introduction}

1 Pour percevoir ce qu'étaient les cultures, l'organisation des sociétés, la place dans l'environnement des hommes de la préhistoire, on se fonde le plus souvent sur des données matérielles tirées du substrat terrestre. Lors de l'exhumation et bien avant l'étude analytique leur position est enregistrée, ceci afin de pouvoir les replacer dans l'espace mais aussi pour leur affecter un âge relatif. Ainsi, elles peuvent être regroupées en ensembles propres à un lieu ou (et) à une période. Le regroupement en ensembles est nécessaire car les particularités présentées par une donnée isolée, quelle qu'en soit l'importance ou l'originalité, n'ont de valeur qu'anecdotique ; ce n'est qu'en menant des recherches sur le groupe que peuvent être mis en évidence les caractères propres à l'ensemble, au groupe en question. La recherche de caractères d'ordre évolutif par exemple n'est possible que si elle concerne des ensembles de données situés dans le temps.

2 Les archives préhistoriques provenant souvent de gisements stratifiés, les moyens offerts par la stratigraphie pour le classement de ces archives ne peuvent être ignorés mais leur utilisation n'est possible que si les ensembles sont regroupés par unité stratigraphique. Celle-ci doit être définie par des caractères bien précisés : d'ordre archéologique pour l'unité archéostratigraphique en archéostratigraphie, d'ordre biologique pour l'unité biostratigraphique en biostratigraphie, d'ordre lithologique pour l'unité lithostratigraphique en lithostratigraphie, d'ordre chronologique pour l'unité chronostratigraphique en chronostratigraphie.... (Hedberg 1979); il existe donc 
autant d'unités stratigraphiques que de branches stratigraphiques, chacune d'elles devant faire l'objet de travaux indépendants dont les résultats sont ensuite confrontés pour répondre aux questions spécifiques qui se posent dans les diverses spécialités. En archéologie préhistorique, le "niveau » ou la «couche " a fait figure, à tort, d'unité stratigraphique car c'est sur la base de caractères d'ordre divers que la tranche de terrain qu'il ou elle représente est différenciée de celle qui est au-dessous comme de celle qui la surmonte.

De toutes les branches de la stratigraphie, c'est la biostratigraphie qui apporte les meilleures précisions de corrélations stratigraphiques. C'est un outil qui permet de situer dans le temps, les unes relativement aux autres, les strates fossilifères. Utilisé de tous temps lors des travaux concernant le domaine marin, cet outil a été adapté au domaine continental il y a seulement quelques décennies par l'établissement de biozonations fondées sur les mammifères des périodes pliocène, pléistocène et holocène (cf. notamment Mein 1975 ; Guérin 1982 ; Cordy 1982 ; Guérin et Patou-Mathis, 1996) ; sa résolution chronologique est suffisamment fine pour dépasser, dans certains cas, celle atteinte par les datations radiochronologiques (Delpech 2005, 2007 ; Delpech et Texier 2007).

De par l'ancienneté des fouilles qui y ont été menées, bien des sites archéologiques «classiques » n'ont pas fait l'objet de telles recherches. C'est sur eux, cependant, que reposent les bases des connaissances des industries préhistoriques. Une révision a été menée pour quelques uns d'entre eux, notamment dans le cadre d'un PCR dirigé par J.Ph. Rigaud puis par J.-P. Texier (Texier et al. 1999; Texier et al. 2004); le site de Laugerie-Haute, quant à lui, a bénéficié pour ses niveaux solutréens d'une campagne de datations, avec croisement des méthodes (Roque et al. 2001), et de recherches géologiques sur leur stratogénèse (Texier 2009). A l'occasion d'un colloque sur le Solutréen qui s'est tenu à Preuilly sur Claise en $2007^{1}$, j'ai revu les questions de biostratigraphie de ce même gisement, m'intéressant, en particulier aux niveaux où ont été définies plusieurs étapes évolutives du technocomplexe solutréen.

\section{« Stratigraphies » à Laugerie-Haute}

5 Situé sur la commune des Eyzies-de-Tayac en Dordogne (fig. 1), le grand abri de Laugerie-Haute s'ouvre en rive droite de la Vézère à deux kilomètres en amont du village. "Il s'étend sur une longueur de 180 mètres environ, sur une largeur de 35 mètres, et l'épaisseur des dépôts varie de 4,50 mètres à 6 mètres suivant les endroits. Une zone non fouillée et qui supporte une maison d'habitation divise le gisement en deux loci: Laugerie-Haute-Est et Laugerie-Haute-Ouest » (Laville 1975 - p. 310).

6 Reconnu comme gisement préhistorique depuis la deuxième moitié du 19ème siècle (Lartet et Christy 1864, 1865-1875 ; Girod et Massénat 1893 ; Capitan 1895 ; Capitan et Breuil 1902 apud Laville 1975), Laugerie-Haute a vu se succéder plusieurs générations de fouilleurs. En 1938 D. et E. Peyrony publient une monographie du site livrant des informations détaillées sur sa stratigraphie. Celle-ci fut reprise et précisée par F. Bordes et par Ph. Smith lors des travaux qu'ils ont menés, à leur tour, il y a une cinquantaine d'années (Bordes 1958 ; Smith 1966). A peu près au même moment, H. Laville, par une étude sédimentologique, établit une « climato-stratigraphie » du site $(1964,1973,1975)$.

7 La mise en regard des propositions stratigraphiques retenues par D. et E. Peyrony et ceux qui leur ont succédé montre non seulement le «souci d'un découpage stratigraphique 
toujours plus fin» (Texier 2007 - p. 49) entraînant la reconnaissance de subdivisions au développement tout à fait local mais aussi des difficultés à synchroniser les relevés de l'est du gisement avec ceux de l'ouest. A l'est du gisement notamment, les 13 ensembles des fouilles Peyrony (couches A à K) correspondent aux 42 ensembles des fouilles Bordes. En outre, selon la zone fouillée, le nombre des couches «non stériles » varie : les cinq couches magdaléniennes $1,2,4,6$ et 8 de l'est du site correspondent sur la coupe II à 8 ensembles (tabl. 1). Quant à la confrontation des successions stratigraphiques de l'est et de l'ouest du gisement, plusieurs remarques et questions ont été soulevées. Sans être exhaustive, rappelons que le niveau Aurignacien V surmonterait le niveau Protomagdalénien (Bordes 1964) et non l'inverse (Peyrony et Peyrony 1938). Les limites entre les ensembles solutréens (Solutréen inférieur, moyen ou supérieur) comme les correspondances entre les ensembles de l'ouest et de l'est ont souvent été difficiles à établir (Bordes 1958; Smith 1966). On peut, en outre, se

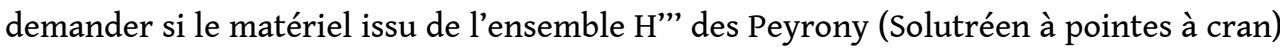
n'était pas, en partie, associé au Magdalénien $0^{2}$, technocomplexe qui, selon $\mathrm{H}$. Laville (1973, 1975), serait contemporain de la période de formation de la couche 0 (côté ouest) (tabl. 1).

Figure 1 - Localisation du grand abri de Laugerie-Haute.

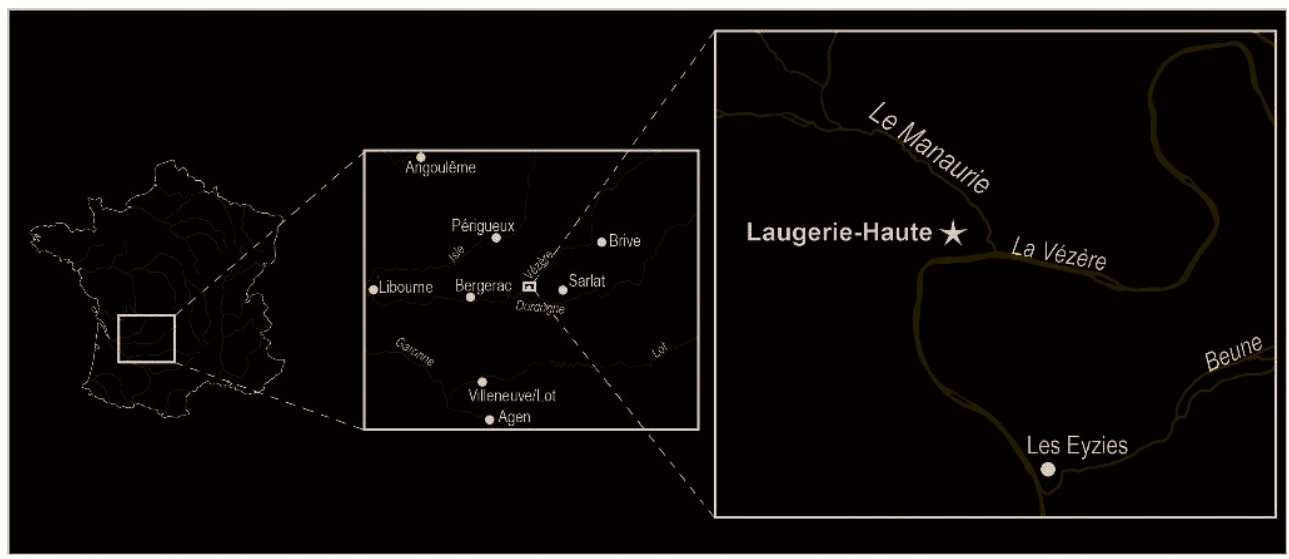


Tableau 1 -Laugerie-Haute-Est et Laugerie-Haute-Ouest (fouilles Peyrony, fouilles Bordes, fouilles Bordes et Smith). Correspondances entre subdivisions stratigraphiques et technocomplexes.

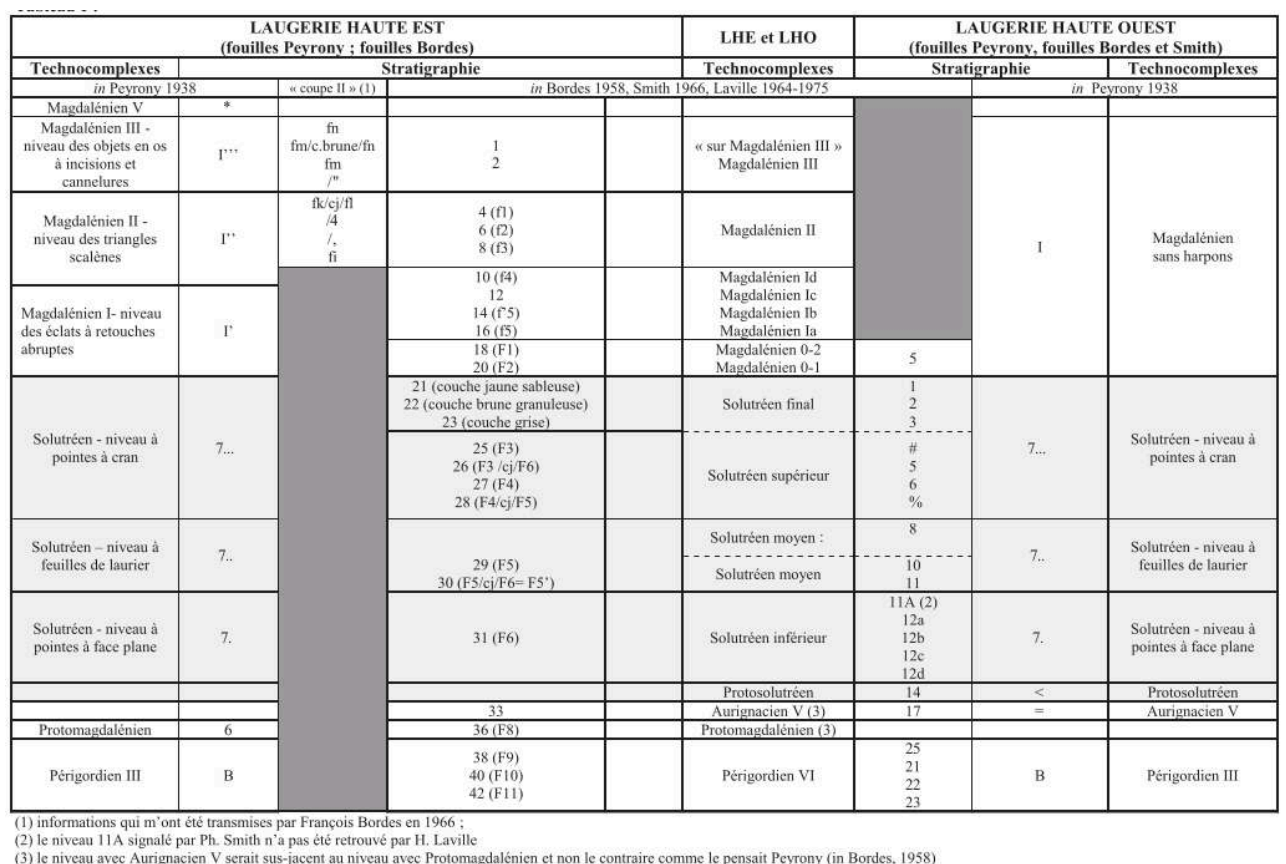

\section{La faune des niveaux solutréens ${ }^{3}$ et « l'Interstade de Laugerie »}

Dans les niveaux solutréens H', H" et H"' (fouilles Peyrony), le Renne est le taxon dominant suivi par le Cheval (beaucoup moins de restes mais toujours signalé en deuxième position) et de quelques ongulés : Cerf, Bouquetin, Bovinés, Mammouth, plus rares que les deux premiers et d'abondance variable selon le niveau concerné. Loup et Renard commun avaient laissé des canines en H"' et l'Ovibos y était représenté par une molaire (déterminations de H.G. Stehlin in Peyrony et Peyrony 1938) ; depuis, ce Bovidé a été identifié en H" (Castel et Madelaine 2006) et l'Antilope saïga a été reconnue en H"' à partir de six vestiges (Madelaine 1989).

Les restes fauniques issus des fouilles Bordes et des fouilles Bordes et Smith menées tant à l'est qu'à l'ouest ont fait l'objet d'un premier examen par F. Prat (in Laville 1964 p. 48 ; Prat 1968) puis d'une étude que j'ai menée dans le cadre de ma thèse (Delpech, 1975, 1983). François Bordes m'avait alors indiqué les correspondances qu'il avait établies, à Laugerie-Haute-Est, entre les couches de la coupe II et celles de la coupe principale (tabl 1). Pour l'anecdote, je précise que c'est sur la base de ces informations que j'ai constitué les trois lots d'ossements qui ont été datés par le radiocarbone à Lyon (Ly 972 pour l'ensemble Magdalénien 0, Ly 973 pour l'ensemble Magdalénien II, Ly 974 pour l'ensemble Magdalénien III). Si les datations du Magdalénien 0 et du Magdalénien III ont été admises sans discussion, celle du Magdalénien II a été rejetée parce que "trop ancienne ». Elle a aussi conduit à suspecter un mauvais travail de collecte de données et à nier l'existence d'un Magdalénien II. 
Tableau 2 - Laugerie-Haute-Est (fouilles Bordes, fouilles Bordes et Smith). Répartition taxinomique par archéostrate du nombre de restes paléontologiques déterminés.

\begin{tabular}{|c|c|c|c|c|c|c|c|c|c|c|c|}
\hline & $\begin{array}{l}\text { Magdal. III } \\
1 \text { et } 2 \\
\text { fn à fl }\end{array}$ & $\begin{array}{c}\text { Magdal. II } \\
4,6 \text { et } 8 \\
\text { fk à fi; } ; \text { fl à f3 }\end{array}$ & $\begin{array}{l}\text { Magdal. I } \\
10 \text { a } 16 \\
f 4 \text { a } 55\end{array}$ & $\begin{array}{l}\text { Magd. } 0 \\
18 \text { et } 20 \\
F 1 \text { et F2 }\end{array}$ & $\begin{array}{c}\text { Solutréen } \\
\text { final } \\
22 \text { et } 23\end{array}$ & $\begin{array}{c}\text { Solutréen } \\
\text { supérieur } \\
25 \text { à } 28\end{array}$ & $\begin{array}{c}\text { Solutréen } \\
\text { moyen } \\
29\end{array}$ & $\begin{array}{c}\text { Solutréen } \\
\text { moyen } \\
30\end{array}$ & $\begin{array}{c}\text { Solutréen } \\
\text { inférieur } \\
31\end{array}$ & $\begin{array}{c}\text { Protomagd. } \\
36\end{array}$ & $\begin{array}{l}\text { Périgor dien VI } \\
36,38 \text { et } 40\end{array}$ \\
\hline \multicolumn{12}{|l|}{ Artiodactyles } \\
\hline Bouquetin & 9 & 3 & 12 & & & 1 & 3 & 2 & 6 & 18 & \\
\hline Bovinés & 17 & 1 & 6 & 7 & & & & 1 & 2 & 2 & \\
\hline Cerf & 13 & 8 & 1 & 4 & & & & & 2 & 33 & 5 \\
\hline Chamois & 12 & 3 & 6 & 22 & & & & & 2 & 9 & 1 \\
\hline Renne & 2143 & 508 & 1188 & 258 & 38 & 134 & 128 & 129 & 319 & 273 & 100 \\
\hline Saïga & 34 & 13 & & 5 & & & & & & & \\
\hline \multicolumn{12}{|l|}{ Perissodactyle } \\
\hline \multirow{2}{*}{\multicolumn{12}{|c|}{$\begin{array}{l}\text { Cheval } \\
\text { Proboscidien }\end{array}$}} \\
\hline & & & & & & & & & & & \\
\hline Mammouth & 1 & & & & & & & & & & \\
\hline
\end{tabular}

Tableau 3 - Laugerie-Haute-Ouest (fouilles Bordes, fouilles Bordes et Smith). Répartition taxinomique par archéostrate du nombre de restes paléontologiques déterminés.

\begin{tabular}{|c|c|c|c|c|c|c|c|c|c|c|c|c|c|c|c|}
\hline \multirow[b]{2}{*}{ Couches } & \multicolumn{2}{|c|}{ Solutréen final } & \multicolumn{4}{|c|}{ Solutréen supérieur } & \multicolumn{2}{|c|}{ Solutréen moyen } & \multicolumn{2}{|c|}{$\begin{array}{c}\text { Solutréen } \\
\text { moyen }\end{array}$} & \multirow{2}{*}{$\begin{array}{c}\begin{array}{c}\text { Solutréen } \\
\text { inférieur inf }\end{array} \\
11 \mathrm{~A}\end{array}$} & \multicolumn{4}{|c|}{ Solutréen inférieur } \\
\hline & 2 & 3 & 4 & 5 & 6 & 7 & 8 & 9 & 10 & 11 & & 12a & $12 \mathrm{~b}$ & $12 \mathrm{c}$ & $12 \mathrm{~d}$ \\
\hline Artiodactyles & & & & & & & & & & & & & & & \\
\hline $\begin{array}{l}\text { Bouquetin } \\
\text { Bovinés }\end{array}$ & 1 & & & & & & 1 & 1 & & & 1 & & 2 & 1 & \\
\hline Cerf & 3 & & & & & & & & & & & & 1 & & \\
\hline Chamois & & & & & & & & & 1 & & & & & & \\
\hline Renne & 236 & 139 & 194 & 432 & 190 & 198 & 199 & 87 & 146 & 5 & 121 & 576 & 509 & 382 & 155 \\
\hline $\begin{array}{l}\text { Périssodactyle } \\
\text { Cheval }\end{array}$ & 3 & 1 & 3 & 2 & 2 & 1 & 4 & & 16 & 2 & 12 & 12 & 15 & 6 & 4 \\
\hline Proboscidien & & & & & & & & & & & & & & & \\
\hline Mammouth & 20 & & & 1 & & & 1 & 21 & 24 & & 11 & 5 & 1 & 2 & 5 \\
\hline $\begin{array}{l}\text { Carnivores } \\
\text { Ours }\end{array}$ & & & 2 & & & & & & & & 2 & & 1 & & \\
\hline Loup & & 1 & 1 & & & & & 1 & & & 1 & & 2 & & \\
\hline Renard & 6 & & & 11 & 1 & 1 & 2 & & 2 & & 1 & 3 & & & \\
\hline Lagomorphe & 3 & 1 & & 3 & & & & & 5 & & & & 1 & & 1 \\
\hline
\end{tabular}

10 Ainsi que D. et E. Peyrony l'avaient indiqué suite aux renseignements fournis par H.G. Stehlin, dans tous les niveaux solutréens le Renne domine fortement suivi par le Cheval beaucoup plus rare. Tous les autres taxons ne sont représentés que par de rares vestiges et de façon épisodique (tabl. 2 et 3). Dans les niveaux 22 à 28 de LaugerieHaute-Est avec Solutréen supérieur et final, F. Prat avait noté une moindre abondance $\mathrm{du}$ Renne que dans les niveaux sous-jacents. Cette baisse en pourcentage du Renne corroborait, selon H Laville $(1973,1975)$, l'interprétation climatique qu'il donnait pour ces mêmes niveaux 22 à 28 à savoir une amélioration assimilée à « l'Interstade Würm III-Würm IV » ou « Interstade de Laugerie » (voir aussi Paquereau 1978). Cette baisse du taux de représentation du Renne ne peut, cependant, être interprétée ainsi. En effet, quand le climat devient plus humide et plus doux, la diminution du Renne va de pair avec une augmentation des ongulés de prairies humides et de forêts. On ne note rien de tel à Laugerie-Haute où c'est le Cheval qui prend de l'importance. Or, cet ongulé s'adapte encore mieux que le Renne à certaines conditions froides et sèches de l'Europe périglaciaire (cf. infra). En outre, la molaire d'Ovibos qui proviendrait aussi de l'ensemble solutréen supérieur H"' (Stehlin in Peyrony et Peyrony 1938; Castel et Madelaine 2006) confère à cet ensemble une note glaciale : l'Ovibos occupe aujourd'hui les zones les plus nordiques de l'hémisphère boréal. Notons aussi que F. Prat puis moimême avions identifié l'Antilope saïga dans les niveaux sus-jacents aux niveaux solutréens, ceux ayant livré à $\mathrm{F}$. Bordes du Magdalénien 0, du Magdalénien II et du Magdalénien III (respectivement niveaux 20 et 18, 8 à 4,2 et 1 de Laugerie-Haute-Est), S. Madelaine l'a identifiée dans l'ensemble solutréen de Laugerie-Haute-Ouest des fouilles Peyrony. Bien qu'elle y ait tout à fait sa place, on ne peut rejeter la possibilité d'une association de ces vestiges au Magdalénien 0 (qui n'avait pas été reconnu par les Peyrony) mais, si l'on admet leur «contemporanéité » avec le Solutréen, ce qui est 
envisageable car une telle association a été rencontrée dans d'autres gisements (Castel et Madelaine 2006), on est alors en possession d'un élément supplémentaire permettant de rejeter l'hypothèse d'un environnement climatique relativement humide : l'Antilope saïga occupe, de nos jours, les zones de steppes arides et les semi-déserts. D'autres éléments encore contribuent à jeter l'hypothèse de «l'Interstade de Laugerie ", l'étude biométrique du Renne notamment. Elle a été menée en 1970 dans le but de tester l'hypothèse de J. Bouchud (1975) selon laquelle, au cours du Paléolithique supérieur, la présence en proportion plus ou moins forte de rennes de toundra et de rennes de forêt aurait expliqué les variations de taille présentées par ce taxon. Il a été montré que ces variations de taille se sont produites au sein d'une même lignée en réponse à des changements environnementaux et que la taille modeste du Renne solutréen de Laugerie comme celle du Renne magdalénien du même site dénotait un environnement particulièrement contraignant (Delpech 1975, 1983, 1986; Delpech et al. 2000). Les travaux sur l'ostéométrie du Renne menés par Delphine Kuntz (2011) confortent cette hypothèse. Ces contraintes sont celles du Dernier Maximum Glaciaire avec, en zone boréale, des déserts froids qui s'étendent, un espace habitable qui diminue et se cloisonne et, de façon générale, des conditions climatiques sévères (Delpech 1999). Il faut aussi rappeler que l'existence d'un "Interstade de Laugerie» (comme d'un "Interstade de Lascaux » postérieur mais très proche dans le temps) (Leroi-Gourhan 1980) a été réfutée par des palynologues dès les années quatre-vingts (Beaulieu et Reille 1984) et plus récemment (Elanga et al. 2000 ; Sanchez Goni 1991, 1994). Il est regrettable que les associations d'ongulés, si caractéristiques du point de vue des environnements, aient servi de caution à l'hypothèse de l'existence d'un interstade, hypothèse qui va à l'opposé de ce que ces associations sont censées argumenter.

\section{Quels changements environnementaux au cours de l'époque solutréenne ${ }^{4}$ ?}

11 Outre l'établissement de conditions climatiques sévères, peut-on percevoir des évolutions, des changements durant la formation de l'ensemble solutréen? Pour ce faire, j'utiliserai les données issues des fouilles François Bordes et Philip Smith: les subdivisions stratigraphiques sont nombreuses et les échantillons fauniques associés ont été déterminés d'un point de vue taxinomique ((et non pas "taxonomique» (Tardieu 2011)). On dispose, en outre, de deux groupes de données, celui de l'est et celui de l'ouest du gisement. D'un côté comme de l'autre, ce sont les mêmes phénomènes environnementaux qui ont présidé à la formation des niveaux archéologiques. Essayons donc de percevoir d'éventuels changements, si changements il y eut, via la recherche de marqueurs tant du côté est que du côté ouest.

Du côté ouest, 14 niveaux livrent suffisamment de matériel pour être pris en considération; du côté est on compte sept niveaux qui ne forment que cinq ensembles car les restes découverts dans les niveaux 22 et 23 d'une part et 25 à 28 d'autre part ont été regroupés avant que nous menions le travail d'identification. Celui-ci (Delpech 1975, 1983) a mis en évidence une majorité de restes d'ongulés (tabl. 2 et 3). Dans les deux locus, c'est le Renne qui domine ; nous l'avons déjà signalé et n'y reviendrons pas ; les différences, si différences il y a, sont donc à chercher ailleurs.

13 À Laugerie-Haute-Est (ou LHE), de la base au sommet, l'évolution des associations d'ongulés apparaît bien ordonnée : on note une augmentation quasi régulière du Cheval 
aux dépens du Renne (tabl. 2, fig. 2a) ce qui, d'un point de vue paléoenvironnemental, était ce que l'on était en droit d'attendre à l'approche du Dernier Maximum Glaciaire : le Cheval est un ongulé très ubiquiste, pouvant s'adapter plus rapidement que tout autre aux conditions les plus diverses, donc les plus sévères. Au Pléistocène supérieur, en Aquitaine qui est une région d'habitat pérenne, il a été présent en permanence tant au cours des périodes les plus froides et sèches qu'aux moments les moins rigoureux. Seul, parmi les ongulés d'Europe, le Cerf aurait pu lui ravir son titre du "plus apte à s'adapter " mais au cours des périodes les plus sévères, le Cheval a toujours mieux réussi que le Cerf : l'effectif de l'espèce caballine a été apparemment toujours plus élevé que celui de ce cervidé ( Delpech et al. 1983 ; Delpech 1999). Au sein de la séquence stratigraphique de LHE, l'ensemble solutréen s'individualise assez nettement de l'ensemble protomagdalénien sous-jacent dans lequel, aux côtés du Renne toujours dominant, le Cheval est très rare tandis que le Cerf et les ongulés de montagne sont relativement bien représentés (fig. 3a) ; au contraire, l'ensemble solutréen ne diffère du premier ensemble magdalénien (niveaux avec Magdalénien 0) qui le surmonte que par la présence de quelques ongulés de montagne et par celle de l'Ovibos représenté par une molaire récoltée lors des fouilles Peyrony. Cette molaire, cependant, comme la sculpture de la tête d'ovibos des déblais Hauser (Peyrony et Peyrony, 1938 - pl. III, fig. 2), pourrait provenir de l'ensemble magdalénien 0 ). Les plus fortes différences apparaissent entre l'ensemble magdalénien 0 et les niveaux magdaléniens sus-jacents où l'on note la rareté du Cheval. Ainsi, à LHE, le développement du Cheval se manifeste comme un processus qui débute dans les niveaux inférieurs avec le Solutréen et s'achève avec le niveau magdalénien 0 .

14 A Laugerie-Haute-Ouest (ou LHO), le Mammouth est assez bien représenté dans les niveaux 11A, 10 et 9 ainsi que dans le niveau 2 au contraire de LHE où il n'a pas été reconnu ${ }^{5}$ (tabl. 3 ; fig. 2b). Ce sont essentiellement des fragments d'incisives ${ }^{6}$ qui lui sont attribuables, ces vestiges pourraient être liés à un travail de l'ivoire qui aurait été mené du côté ouest du site. D'un point de vue environnemental, leur présence indique l'établissement d'un climat froid permettant cependant le développement d'une biomasse végétale suffisamment forte pour nourrir le plus grand des ongulés pléistocènes. Toujours du côté ouest, on note une augmentation relativement forte de la représentation du Cheval dans les niveaux 11A et 10 c'est- à- dire dans les niveaux ayant livré du Solutréen inférieur et du Solutréen moyen. On ne retrouve pas l'augmentation graduelle constatée à l'est du site qui s'y présente comme un processus évolutif débutant à la base de la séquence solutréenne et se poursuivant jusqu'à son sommet. Comment alors établir des liens entre la séquence solutréenne de l'est et celle de l'ouest du site? Traitons cette question avec le point de vue du biostratigraphe. 
Figure 2 - Distribution des ongulés dans les niveaux solutréens.

2a, à Laugerie-Haute-Est ;

$2 b$ à Laugerie-Haute-Ouest (fouilles Bordes, fouilles Bordes et Smith).

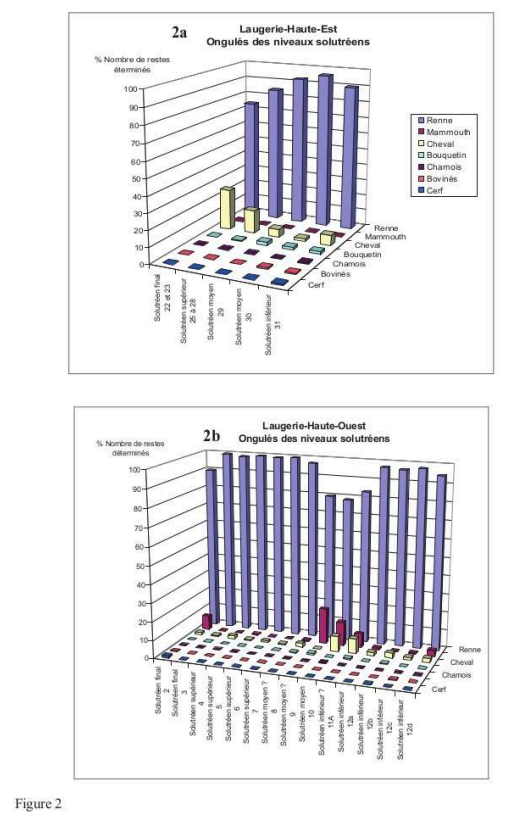

\section{Unités biostratigraphiques des ensembles solutréens et chronologie}

15 Pour définir ces unités, nous avons considéré l'ensemble des séquences stratigraphiques du gisement qui, à LHO, concernent le Solutréen et, à LHE, le Périgordien VI, le Protomagdalénien, le Solutréen et le Magdalénien. Ces unités ont été établies suivant les principes développés dans les travaux de divers auteurs (cf. notamment Guérin 1982; Cordy 1982), à savoir en considérant les taxons caractéristiques des lignées guides, les associations d'ongulés et les espèces caractéristiques. S'agissant d'une séquence formée lors d'un court moment du Pléistocène, on peut s'attendre à ce que les taxons caractéristiques de lignées-guides soient de peu d'utilité ici (Delpech 2007). En effet, ces taxons, Equus caballus gallicus (Prat 1968) pour le Cheval et pour le Renne une forme de petite taille (Delpech 1975, 1986 ; Delpech et al. 2000 ; Kuntz 2011), sont présents tout au long de la séquence ; ils n'apportent donc aucun élément de discrimination des niveaux en question.

À LHE, nous avons identifié 5 unités biostratigraphiques (fig. 3a); l'une d'elles regroupe trois niveaux qui contiennent des associations peu différentes (cas de l'unité A correspondant aux niveaux avec Magdalénien III, II et I) ; trois autres correspondent, chacune, à un seul niveau qui livre une association particulière (cas de l'unité $\mathrm{B}, \mathrm{D}$ et $\mathrm{E}$ avec respectivement du Magdalénien 0, du Protomagdalénien et du Périgordien VI) ; la dernière unité (unité $\mathrm{C}$ ) regroupe tous les niveaux avec Solutréen; chacun d'eux livre une association un peu différente de l'autre mais, d'un niveau à l'autre, l'augmentation 
du Cheval est progressive et assez régulière, le tout allant de pair avec une diminution de la diversité : le Cerf et les Bovinés disparaissent en premier, suivis par le Bouquetin. De la base au sommet, les unités biostratigraphiques de LHE permettent de retracer une évolution environnementale logique (cf. § précédent). Dans cette séquence s'intègre l'unité solutréenne formée de cinq ensembles superposés pour lesquels aucun argument d'ordre biostratigraphique ne vient remettre en cause la succession chronologique.

17 À LHO, tous les niveaux livrent du Solutréen. Du point de vue de la composition des associations fauniques, ces niveaux se différencient de ceux de l'est, notamment, par la présence du Mammouth. Ce fait ne pourra, cependant, pas être utilisé pour établir des corrélations entre LHE et LHO puisqu'il s'agit d'une originalité propre à LHO. D'un point de vue biostratigraphique, cinq unités, dénommées C, peuvent être retenues (fig. 3b). L'unité C3 se caractérise par la présence du Mammouth. Dans deux autres unités, C5 et C2, le Renne laisse très peu de place aux autres ongulés.; ceux-ci sont surtout représentés par le Cheval; les variations de leur taux de représentation, bien que faibles, y apparaissent tout à fait aléatoires. On remarque toutefois la présence dans l'unité C5 du Cerf (niveau 12b) et des Bovinés (niveau 12c) ce qui permet de tracer un lien entre les deux locus : à LHO, comme à LHE, ces deux taxons sont représentés à la base de l'ensemble stratigraphique livrant du Solutréen. L'unité $\mathrm{C} 1$, qui correspond au seul niveau 2 sommital, se caractérise aussi par une assez forte richesse taxinomique (nombre d'ongulés $=5$ ) et par la présence du Cheval, de Bovinés et du Cerf comme dans l'unité de base C5 (niveau 12b) de LHO et comme à la base de l'unité C de LHE. On est donc conduit à tisser un lien entre les unités $\mathrm{C} 1$ et $\mathrm{C} 5$ de LHO d'une part, et la base de l'unité $\mathrm{C}$ de LHE d'autre part. Enfin, l'unité $\mathrm{C} 4$ se caractérise par une augmentation, pas très forte mais nette, du Cheval (puis du Mammouth). Rappelons que, à LHE dans le Solutréen, c'est la montée en puissance du Cheval qui justifie l'ordonnancement des niveaux archéologiques; à LHO, c'est dans l'unité C4 que s'élève le taux de représentation du Cheval (cf. fig. $2 b$ et fig. $3 b$ ).

Les corrélations établies entre LHE et LHO montrent clairement que les unités de LHO ne sont pas disposées selon un ordre chronologique normal. En effet, à la formation de l'unité $\mathrm{C}$ de l'est (niveaux 31 à 22) correspondent successivement à l'ouest les unités C5 (niveau 12) et C1 (niveau 2), puis l'unité C2 (niveaux 8 à 3) et enfin l'unité C4 (niveaux 10 et $11 \mathrm{~A}$ ) qui serait la plus récente. Le rapprochement dans le temps des périodes de formation des niveaux 12 et 2 serait presque suggéré par les datations : à LHO, les dates les plus anciennes des niveaux livrant du Solutréen supérieur et final sont celles de la couche 2 (fig. 4b). Toutefois, face à de tels bouleversements justifiés par des variations de taux de représentation somme toute relativement faibles, il paraît plus raisonnable, de regrouper l'ensemble des niveaux solutréens de LHO dans une même unité biostratigraphique. 
Figure 3 - Unités biostratigraphiques. 3a, à Laugerie-Haute-Est ; 3b, à Laugerie-Haute-Ouest (fouilles Bordes, fouilles Bordes et Smith).
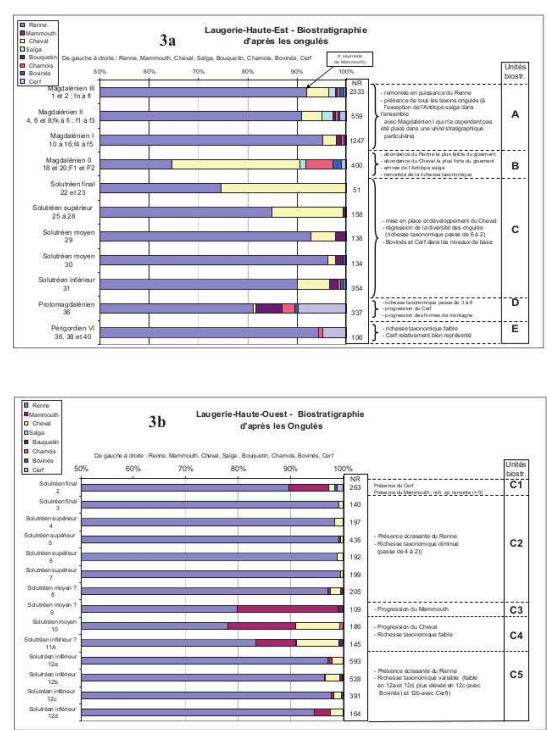

Figure 3

Figure 4 - Chronologie ${ }^{14} \mathrm{C}$ à Laugerie-Haute (années cal BP). 4a, chronologie de l'ensemble de la séquence archéologique ; $4 \mathrm{~b}$, chronologie des stades culturels du Solutréen.
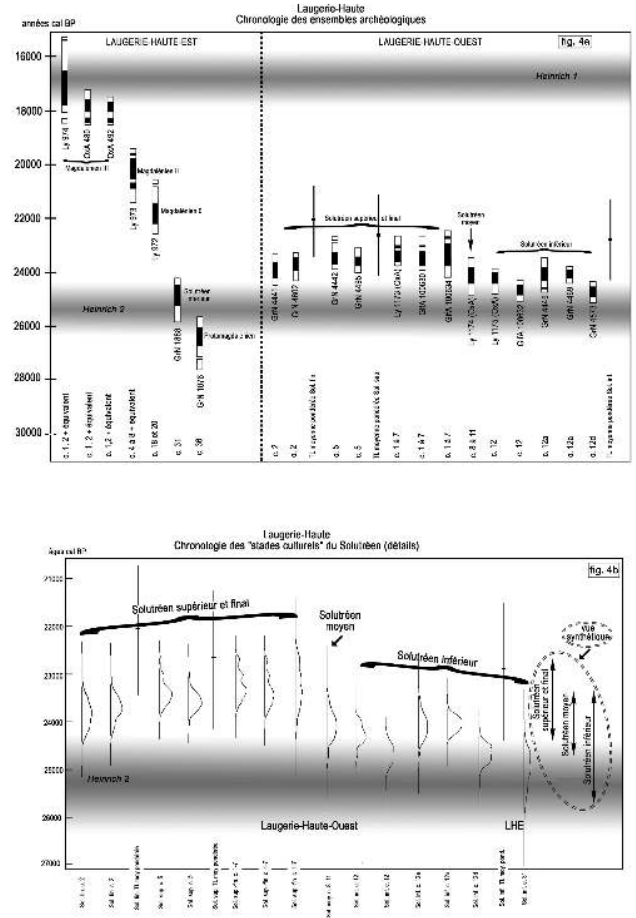


\section{Chronologie et datations}

19 Ainsi, la biostratigraphie conduit à valider l'ordre chronologique des niveaux solutréens 31 à 22 de LHE et à ne pas retenir celui des niveaux 12 à 2 de LHO. Ceux-ci devraient donc être considérés globalement pour tous travaux nécessitant de s'appuyer sur des données classées dans un ordre chronologique.

D'autres travaux menés sur les niveaux solutréens de l'ouest du gisement ont conduit à des conclusions qui soutiennent ces points de vue. Ainsi, la mise en place des dépôts solutréens apparaît clairement dominée par la solifluxion qui peut occasionner la formation de "pseudo-niveaux stratifiés » à partir d'un même niveau archéologique «et provoquer d'importantes perturbations de l'ordre originel des nappes de vestiges» (Texier 2009 - p. 140). Par ailleurs, dans le cadre d'une expérience de croisement de datations TL/ $14 \mathrm{C}$, «le recouvrement des résultats relatifs aux différents niveaux stratigraphiques montre que le découpage ancien de la séquence de Laugerie-Haute " (côté ouest) "réalisé sur la base de l'outillage n'est pas associé à des écarts chronologiques significatifs.... Dans l'utilisation des dates, les préhistoriens doivent désormais prendre en compte le "flou » résultant de l'ensemble des incertitudes physiques de la datation" (Roque et al. 2001 - p. 231, fig. 6). La figure 4 qui réunit toutes les datations radiocarbones obtenues à l'est et à l'ouest du gisement, comme les dates TL de l'ouest, éclaire en ce sens. Dans l'ensemble, les datations sont cohérentes : de la base au sommet de la séquence on monte dans la chronologie (fig. 4a) mais, côté ouest, pour le Solutréen seul, il est impossible de donner un âge propre à chaque «étape » du technocomplexe (fig. $4 \mathrm{~b}$ ). Notons que, relativement aux périodes de grandes débacles d'icebergs, la séquence solutréenne de Laugerie-Haute se serait formée postérieurement à l'événement Heinrich 2 qui aurait duré de l'ordre de 2,2 millénaires et aurait débuté il y a près de 26400 ans (Sanchez-Goni M.F. et Harrison S.P. 2010).

\section{Conclusion}

21 A Laugerie-Haute, la faune associée au Solutréen caractérise un climat globalement froid et sec. C'est une interprétation déjà formulée en 1975 (Delpech 1975 - p. 296, 1983 - p. 279) qui, alors, était tout à fait opposée à celle d'un radoucissement ayant valeur d'interstade soutenue par des palynologues et sédimentologue (Paquereau 1978 ; LeroiGourhan 1980; Laville 1964, 1973 et 1975). En cela, ces auteurs suivaient D. et E. Peyrony qui pensaient qu'un radoucissement s'était produit au cours du Solutréen (Peyrony et Peyrony 1938 - p. 77). Aujourd'hui, le Solutréen est devenu définitivement " froid ». Ce travail en réunit quelques arguments paléontologiques. En outre, quand on monte dans la séquence stratigraphique de Laugerie-Haute-Est, tout indique une gradation de la sévérité du climat comme on pouvait s'y attendre à l'approche du Dernier Maximum Glaciaire, le maximum de sévérité ayant été atteint alors que se formait l'ensemble avec Magdalénien 0. Cette progression graduelle soutient l'hypothèse selon laquelle, à Laugerie-Haute-Est, les niveaux solutréens fossilifères sont des entités stratigraphiques ordonnées dans le temps. Mais cette progression n'est visible qu'à l'est du site; à l'ouest on ne trouve pas trace d'évolution graduelle. La biostratigraphie montre qu'il y a, de plus, désorganisation chronologique des niveaux rendant leur regroupement indispensable pour tous travaux fondés sur des données classées dans le temps. D'autres auteurs, dans d'autres domaines de recherche, incitent 
à prendre ces mêmes précautions (Roque et al. 2001 ; Texier et al. 1999 ; Texier et al., 2004; Texier 2009). Une révision archéostratigraphique serait, nous semble-t-il, maintenant nécessaire. C'est à Laugerie-Haute que l'industrie solutréenne a été caractérisée, que les différentes phases du Solutréen ont été définies. Ph. Smith présente Laugerie-Haute comme étant «le pilier central de la reconstitution du Solutréen français» (Smith 1966 - p. 57). Pour que ce pilier résiste aux assauts du temps, il serait bien qu'il soit soutenu par un travail d'archéostratigraphie plaçant ses bases chronologiques plus nettement à l'est qu'à l'ouest du site. Mais c'est là le point de vue du seul biostratigraphe!

\section{BIBLIOGRAPHIE}

BEAULIEU J.L. (de) ,et REILLE M. 1984 - A long Upper Pleistocene pollen record from Les Echets, near Lyon, France. Boreas, 13, 2, p. 111-132 .

BORDES F. 1958 - Nouvelles fouilles à Laugerie-Haute-Est. Premiers résultats. L'Anthropologie, 62, p. 205-244.

BOUCHUD J. 1975 - Essai sur le Renne et la climatologie du Paléolithique supérieur. Imprimerie Magne, Périgueux

CASTEL J.-C. et MADELAINE S. 2006 - Quelques éléments remarquables de la faune du Solutréen de Laugerie-Haute (Les Eyzies-de-Tayac, Dordogne). Paleo, 18 p. 275-284.

CORDY J.-M. 1982 - Biozonation du Quaternaire postvillafranchien continental d'Europe occidentale à partir des grands mammifères. Annales de la Société géologique de Belgique (Liège), 105 p. 303-314.

DELPECH F. 1975 - Les faunes du Paléolithique supérieur dans le Sud-Ouest de la France. Thèse de Doctorat ès Sciences naturelles, $n^{\circ} 479,374 \mathrm{p}$.

DELPECH F. 1983 - Les faunes du Paléolithique supérieur dans le Sud-Ouest de la France. Cahiers du Quaternaire, Editions du CNRS, $\mathrm{n}^{\circ} 6,453 \mathrm{p}$.

DELPECH F. 1986 - Les rennes du grand abi de Laugerie-Haute en Dordogne (fouilles F. Bordes). Arqueologia, Porto, 13 p. 66-71.

DELPECH F. 1999 - Biomasse d'Ongulés au Paléolithique et inférences démographiques. Paleo, vol. 11 - p. 19-42.

DELPECH F. 2005 - Utilité et utilisation de la biostratigraphie en archéologie préhistorique. Bulletin de la Société préhistorique française, t. 102, 4 p. 749-755.

DELPECH F. 2007- Le grand abri de La Ferrassie, source de réflexion sur la biostratigraphie d'un court moment du Pléistocène. In R. Desbrosse et A. Thévenin coords. Arts et cultures de la préhistoire, Paris, Éd. du CTHS, Documents préhistoriques, 24, p. 303-314.

DELPECH F., DONARD E., GILBERT A., GUADELLI J.-L., LE GALL O., MARTINI-JACQUIN A., PAQUEREAU M.-M., PRAT F, TOURNEPICHE J.-F. 1983 - Contribution à la lecture des paléoclimats 
quaternaires d'après les données de la paléontologie en milieu continental. In Paléoclimats, Bulletin de l'Institut géologique du Bassin d'Aquitaine, 34 et Cahiers du Quaternaire, nº spécial p. 165-177.

DELPECH F., GRAYSON D.K., RIGAUD J.-Ph. 2000 - Biostratigraphie et paléoenvironnements du début du Würm récent d'après les grands mammifères de l'abri du Flageolet I (Dordogne, France). Paleo, 12, p. 97-126.

DELPECH F., TEXIER J.-P. 2007 - Approche stratigraphique des temps gravettiens : l'éclairage aquitain. In Rigaud J.Ph. (ed.), Le Gravettien : entités régionales d'une paléoculture européenne, Table Ronde - Les Eyzies - juillet 2004, Paleo, 19, p. 15-29.

ELANGA H., PEYRON 0., BONNEFILLE R., JOLLY D., CHEDDADI R., GUIOT J., ANDRIEU V., BOTTEMA S., BUCHET G., BEAULIEU J. (de), HAMILTON A.C., MALEY J., MARCHANT R., PEREZ-OBIOL R., REILLE M., RIOLLET G., SCOTT L., STRAKA H., TAYLOR D., VAN CAMPO E., VINCENS A., LAARIF F., JONSON H. 2000 - Pollen-based biomer reconstruction for southern Europe and Africa 18,000 yr BP. Journal of Biogeography, 27, p. 621-634.

GUÉRIN C. 1982 - Première biozonation du Pléistocène européen, principal résultat biostratigraphique de l'étude des Rhinocerotidae (Mammalia, Perissodactyla), du Miocène terminal au Pléistocène supérieur d'Europe occidentale. Geobios, 15, 4, p. 593-598.

GUÉRIN C., PATOU-MATHIS M. 1996 - Limites et problèmes de chronologie. In : Guérin, Cl. ; Patou-Mathis, M.(eds), Les grands mammifères plio-pléistocènes d'Europe, Paris, Masson (Collection préhistoire) p. 1-11.

HEDBERG H. 1979 - Guide stratigraphique international. Classification, terminologie et règles de procédure. Paris, Doin, 233 p.

KUNTZ D. 2011 - Ostéométrie et migration(s) du Renne (Rangifer tarandus) dans le Sud-Ouest de la France au cours du dernier Pléniglaciaire et du Tardiglaciaire (21500-13000 cal. BP). Thèse de Doctorat en Préhistoire, Université de Toulouse 2 Le Mirail, 476 p.

LAVILLE H. 1964 - Recherches sédimentologiques sur la paléoclimatologie du Würmien récent en Périgord. L'Anthropologie (Paris), t. 68, p. 1-48 ; p. 219-252.

LAVILLE H. 1973 -. Climatologie et chronologie du Paléolithique en Périgord. Etude sédimentologique de dépôts en grotte et sous abris. Thèse de doctorat ès Sciences naturelles. Bordeaux, $n^{\circ}$ 400, $720 \mathrm{p}$.

LAVILLE H. 1975 - Climatologie et chronologie du Paléolithique en Périgord : étude sédimentologique de dépôts en grotte et sous abris. Etudes quaternaires, mém. $\mathrm{n}^{\circ} 4$, Université de Provence, $422 \mathrm{p}$.

LEROI-GOURHAN Arl. 1980 - Interstades würmiens : Laugerie et Lascaux. Bulletin de l'Association française pour l'Etude du Quaternaire, 3, p. 95-100.

MADELAINE S. 1989 - Contribution des anciennes fouilles à la connaissance des ongulés et de leurs milieux durant le Würm récent en Dordogne. Paleo, 1, p. 3646.

MEIN P. 1975 - Résultats du groupe de travail des vertébrés. Report on activity on the RCMNS working groups (1971-1975). Bratislava, UGS regional committee on Mediterranean Neogene stratigraphy, p. 78-81.

PAQUEREAU M.-M. 1978 - Flores et climats du Würm III dans le Sud-Ouest de la France. Quaternaria, Roma, XX, p. 123-164.

PEYRONY D. et PEYRONY E. 1938 - Laugerie-Haute près des Eyzies (Dordogne). Archives de l'Institut de Paléontologie Humaine, 19, 84 p. 
PRAT, F. 1968 - Recherches sur les Équidés pléistocènes en France. Thèse de doctorat ès Sciences naturelles. Bordeaux $n^{\circ} 226,622 \mathrm{p}$.

ROQUE C., GUIBERT P., VARTANIAN E., BECHTEL F., OBERLIN C., EVIN J., MERCIER N., VALLADAS H., TEXIER J.-P., RIGAUD J.-PH., DELPECH F., CLEYET-MERLE J.-J., TURQ A. 2001 - Une expérience de croisement de datations TL/14C pour la séquence solutréenne de Laugerie-Haute, Dordogne, in Barrandon J.-N., Guibert P., Michel V. (éds) Datation, XXIe Rencontres Internationales d'Archéologie et d'Histoire d'Antibes, Editions APDCA, Antibes, p. 217-232.

SANCHEZ GONI M.F. 1991 - On the Last Glacial Maximum and interstadials during the Solutrean : a contradiction ? Current Anthropology, 32, 5, p. 573-575.

SANCHEZ GONI M.F. 1994 - The identification of European Upper Palaeolithic interstadials from cave sequences. AASP Contributions Series, 29, p. 161-182.

SANCHEZ-GONI M.F., HARRISON S.P. 2010 - Millenial-scale climate variability and vegétation changes during the Last Glacial : concepts and terminology. Quaternary Science Reviews, 29, p. 2823-2827.

SMITH P.E.L. 1966 - Le Solutréen en France. Bordeaux (France) Delmas, publications de l'Institut de Préhistoire de l'Universié de Bordeaux, 4, 449 p.

TARDIEU C. 2011 - La bonne orthographe du mot Taxinomie : un concept important dont l'orthographe est malmenée. Paleo, n²2, p. 331-334.

TEXIER J.-P. 2007 - Anciennes et nouvelles lectures géologiques de sites paléolithiques de référence du Périgord : évolution des concepts. In «Un siècle de construction du discours scientifique en Préhistoire ", actes du 26ème congrès préhistorique de France, Avignon, 21-25 septembre 2004, p. 47-53.

TEXIER J.-P. 2009 - Histoire géologique de sites préhistoriques classiques du Périgord : une vision réactualisée. Documents préhistoriques n² 25, éditions du CTHS, Paris 2009, 193 p.

TEXIER J.-P., DELPECH F., RIGAUD J. Ph. 1999 - Rapport final de synthèse du PCR : « Révision litho- et biostratigraphique de quelques sites de références périgourdins », Bordeaux, SRA Aquitaine.

TEXIER J.-P., KERVAZO B., LENOBLE A., NESPOULET R. 2004 - Sédimentogénèse de sites préhistoriques du Périgord. Association des Sédimentologistes Français (éd.), 69 p.

\section{NOTES}

1. «Le Solutréen, 40 ans après Smith 66 ", 28-31 octobre 2007, organisateur : association SERAP Vallée de la Claise.

2. J'ai conservé ici la dénomination "Magdalénien » utilisée à l'époque des fouilles (et après) ; aujourd'hui, on parle de « Badegoulien ».

3. L'ensemble protosolutréen (couche $G$ des fouilles Peyrony, couche 14 des fouilles Bordes) n'a livré que très peu de restes de faune ; il n'en sera pas question ici.

4. Le Solutréen n'étant pas une "période" mais un technocomplexe, l'époque solutréenne considérée ici est celle au cours de laquelle se sont formés les "niveaux" solutréens de LaugerieHaute.

5. Nous ne l'avons identifié que dans l'ensemble avec Magdalénien III à partir d'un fragment de molaire. 
6. Tous les restes attribués au Mammouth $(n=91)$ provenant de l'ouest du site sont des fragments d'incisives (de l'ivoire) à l'exception de 14 d'entre eux qui sont des fragments de molaires (1 en 5, 1 en 9,11 en 10 et 1 en $12 \mathrm{c})$.

\section{RÉSUMÉS}

En archéologie préhistorique, la biostratigraphie sert à établir des liens chronologiques entre strates de gisements différents ainsi qu'à mettre en évidence l'existence d'éventuelles perturbations dans l'agencement des strates d'un même site. L'étude menée ici concerne les niveaux solutréens de Laugerie-Haute (Les Eyzies, Dordogne). Il est d'abord question de la stratigraphie globale du site puis de la signification environnementale des associations fauniques et du rôle qu'elles ont joué dans le soutien du «légendaire Interstade de Laugerie». Les recherches d'ordre biostratigraphique conduisent ensuite à valider l'ordre chronologique des niveaux solutréens 31 à 22 qui se développent à Laugerie-Haute-Est et à réfuter celui des niveaux 12 à 2 de Laugerie-Haute-Ouest. Dorénavant, ces derniers devraient être considérés dans leur ensemble lors de toutes recherches nécessitant de s'appuyer sur des données classées dans le temps. Ce travail suggère, en outre, l'intérêt qu'il y aurait à réviser l'archéostratigraphie du Solutréen de Laugerie-Haute, en s'appuyant sur les données les mieux classées chronologiquement : celles de Laugerie-Haute-Est.

Biostratigraphic analysis applied to prehistoric archaeological contexts can establish chronological ties between strata at different sites and document stratigraphic difficulties within a given site. Here, I provide such an analysis for the Solutrean levels of Laugerie-Haute (Les Eyzies, Dordogne). I first address the stratigraphy of the site as a whole and then the environmental significance of the faunal associations found here, including the role that these associations have played in supporting the reality of the famous Laugerie Interstade. The results of this work support the chronological order of Solutrean levels 31 to 22 at Laugerie-Haute-Est but reject that of levels 12 to 2 at Laugerie-Haute-Ouest. These latter levels should be considered as a single unit in all future chronological analyses. As a result, archaeostratigraphic analyses of the Solutrean should be based only on the sequence provided by Laugerie-Haute-Est.

\section{INDEX}

Mots-clés : Laugerie-Haute-Est, Laugerie-Haute-Ouest, unités biostratigraphiques, unités archéostratigraphiques, chronologie du Solutréen

Keywords : Laugerie-Haute-Est, Laugerie-Haute-Ouest, Solutrean, biostratigraphic units, archeostratigraphic units, Solutrean chronology

\section{AUTEUR}

\section{FRANÇOISE DELPECH}

Université Bordeaux 1, PACEA-PPP-UMR 5199, Bâtiment B18, avenue des Facultés, 33405 Talence, France - francoise67.delpech@orange.fr 